\title{
Comparação e mapeamento de grupos municipais do estado do Rio Grande do Sul a partir de indicadores socioeconômicos
}

\author{
Comparison and mapping of municipal groups in the state of Rio Grande do Sul \\ based on socioeconomic indicators
}

\author{
Mariana Motta Dias da Silva', Augusto Maciel da Silva", Enio Junior Seidel'", \\ Ana Lucia Souza Silva Mateus'v, Angela Pellegrin Ansujv
}

\begin{abstract}
RESUMO
Entender como determinadas regiões se comportam e/ou se assemelham em aspectos socioeconômicos pode contribuir para o desenvolvimento regional. Desta forma, este trabalho tem por objetivo identificar semelhanças de comportamento socioespacial existentes entre os municípios do estado do Rio Grande do Sul, a partir dos dados censitários, e avaliar as mudanças ocorridas com o passar dos anos. Para tal foi realizada uma análise de agrupamento dos municípios perante alguns indicadores. Com os agrupamentos verificou-se a existência de diferença no comportamento entre os grupos através de testes não paramétricos e também foram criados mapas temáticos que trazem de maneira espacial o comportamento das regiões. Foram detectados cinco agrupamentos de municípios em 1991 e quatro agrupamentos nos anos de 2000 e 2010. Também é perceptível o desenvolvimento socioespacial com o passar dos anos. Entretanto em 2010 é quando ocorre a maior evolução para a maioria dos municípios. A partir dos agrupamentos gerados é possível traçar estratégias de políticas públicas e ainda priorizar as medidas que devem ser tomadas, pelos órgãos competentes, em cada uma das regiões estudadas.
\end{abstract}

Palavras-chave: Análise de agrupamentos; Comparação de grupos; Mapas temáticos; Grupos regionais.

\begin{abstract}
Understanding how particular regions behave and / or resemble socioeconomic aspects can contribute to regional development. Thus, this paper aims to identify similarities of socio-spatial behavior existing among the municipalities of the state of Rio Grande do Sul, based on census data, and to evaluate the changes that have occurred over the years. For this, a cluster analysis of the municipalities was performed before some indicators. With the clusters was verified the existence of difference in the behavior between the groups through non-parametric tests and thematic maps were created that bring the behavior of the regions in a spatial way. Five clusters of municipalities were detected in 1991 and four clusters in the years 2000 and 2010. It is also noticeable the socio-spatial development over the years. However in 2010 is when the greatest evolution occurs for most municipalities. From the generated clusters it is possible to outline public policy strategies and further prioritize the measures that should be taken by the competent bodies in each of the regions studied.
\end{abstract}

Keywords: Cluster analysis; Group comparison; Thematic maps; Regional groups. 


\section{INTRODUÇÃO}

A forma como ocorre a evolução de uma localidade pressupõe a ocorrência de políticas públicas efetivas, onde se tem de fato o conhecimento da mesma e uma elaboração planejada de ações que tenham grande capacidade de êxito (TRENNEPOHL E PAIVA, 2012).

Uma maneira de representar e analisar a situação vivenciada por um local ou região em determinada época, bem como a evolução desta no tempo e/ou no espaço pode ser realizada por meio da utilização de métodos estatísticos, estatísticas públicas e indicadores sociais e econômicos. Para Jannuzzi (2012), o indicador social se transforma em uma ferramenta funcional, no acompanhamento da situação social, possibilitando criar ou modificar políticas públicas.

A utilização das informações e resultados estatísticos, a definição de metas e o direcionamento e priorização de intervenções, agilizam o processo e eficiência dos gestores públicos para alcançarem os seus objetivos. Assim, os indicadores sociais são desenvolvidos com a finalidade de compreender a realidade social do todo que é analisado (PONTES, 2018).

Ferramentas de estatística têm se mostrado de grande utilização no estudo dos indicadores em distintas abrangências territoriais, como em cidades, estados, regiões e grupos de países. Auxiliando na identificação de similaridades entre regiões ou comparação de comportamentos socioeconômicos. Cita-se como estudos, Crispim, Fernandes e Albuquerque (2019), que utilizaram Análise de Agrupamento no estudo de indicadores de sustentabilidade nos municípios do Marajó-PA. Em Castro, Miranda e Lima (2015) é realizada uma pesquisa sobre indicadores sociais de desenvolvimento e a produção de soja nos 150 maiores municípios produtores brasileiros, utilizando Análise Fatorial. Ainda, de forma mais ampla, Leite, Brigatte e Aguilar (2009) utilizaram metodologias de agrupamento afim de verificar semelhanças entre os países do G-20 perante indicadores socioeconômicos. Em relação ao Rio Grande do Sul, as desigualdades físico territoriais não são as únicas, já que também ocorrem grandes desigualdades econômicas e sociais entre os municípios (CONCHA, 2013). Schneider e Waquil (2001) realizaram um estudo para verificar as diferenças entre os municípios e 
a maneira como a versatilidade das regiões influenciavam, sendo este um dos primeiros estudos referente aos municípios riograndenses. Alonso, Benetti e Bandeira (1984), em seu estudo concluíram que o estado tem ao menos três atividades regionais distintas. Contudo, ainda são necessários estudos que considerem uma maior gama de características sociais ou socioeconômicas para uma melhor descrição dos comportamentos existentes no estado.

Avaliar a situação do estado do Rio Grande do Sul, pode contribuir para o processo de desenvolvimento regional e é de grande valor retratar o comportamento social, sua evolução e estruturação. Perante a carência de trabalhos que tratem dos aspectos regionais sobre o enfoque dos indicadores utilizados, a concepção desse estudo tem seu ineditismo ao procurar identificar padrões do comportamento social através de análise de agrupamento e posterior aplicação de testes não-paramétricos para a comparação dos grupos gerados.

O objetivo deste estudo é avaliar agrupamentos de municípios do estado do Rio Grande do Sul, nos censos de 1991, 2000 e 2010, com base em indicadores socioeconômicos. Através da elaboração de mapas temáticos, busca-se o entendimento de regiões similares, respondendo ao questionamento de como elas se distribuem ao longo dos diferentes censos analisados e se os grupos são estatisticamente distintos quanto aos indicadores utilizados. Além disso, realiza-se a caracterização destes grupos para compreensão da dinâmica socioeconômica do estado.

\section{MATERIAIS E MÉTODOS}

Para realizar o estudo do comportamento dos municípios do Rio Grande do Sul, tendo como base Jannuzzi (2012) os indicadores selecionados foram: Taxa de mortalidade infantil, Esperança de vida ao nascer, Taxa de analfabetismo, Expectativa de anos de estudo, Taxa de atividade, Razão de dependência, Taxa de desocupação, Rendimento médio do trabalho, Renda ou PIB per capita, Parcela de renda apropriada pelos 20\% mais pobres e Índice de Desenvolvimento Humano.

Posteriormente, foram obtidos os indicadores na plataforma "Atlas do Desenvolvimento Humano no Brasil" (Programa das Nações Unidas para o 
Desenvolvimento, PNUD, Instituto de Pesquisa Econômica Aplicada - Ipea e Fundação João Pinheiro - FJP), para todos os municípios do estado do Rio Grande do Sul. Os indicadores Taxa de atividade, Taxa de desocupação e Rendimento médio do trabalho não apresentaram informações no censo de 1991, optando-se por retirá-los da análise. Após identificação dos municípios que existiam e possuíam informações completas nos censos de 1991, 2000 e 2010, totalizou-se 496 municípios para a análise.

Inicialmente, foi aplicada a análise de agrupamento dos dados, para apontar a similaridade e o agrupamento de municípios, por meio da distância euclidiana. A escolha do método de agrupamento se deu por meio da comparação entre cinco métodos: o método de Ward; método das médias; método do centroide; método do vizinho mais próximo; e o método do vizinho mais distante (SILVA et al., 2019).

Como indicação da qualidade dos agrupamentos foi utilizado o coeficiente de correlação cofenética (CCC). A partir do CCC optou-se pelo método das médias. Detalhes da aplicação da CCC nos dados podem ser obtidos em Silva et al. (2019), que mostraram que para a situação em estudo, o maior coeficiente de correlação cofenética encontrado foi para o método das médias, garantindo assim um melhor agrupamento. O método utiliza as médias aritméticas das distâncias entre todos os pares indivíduos que estão em dois grupos distintos. É definido como:

$$
d\left(G_{1}, G_{2}\right)=\sum_{l \in G_{1}} \sum_{k \in G_{2}} \frac{d\left(X_{1}, X_{2}\right)}{n_{1} n_{2}}
$$

em que $n_{1}$ e $n_{2}$ são, respectivamente, os números de objetos dos grupos $G_{1}$ e $G_{2}$ e $d\left(X_{1}, X_{2}\right)$ as distâncias entres todos os pares de elementos que estão presentes nos dois grupos.

A partir dos grupos obtidos no estudo de Silva et al. (2019), são realizadas comparações não paramétricas entre estes grupos por meio do teste de Kruskal-Wallis e post hoc por meio do teste de Dunn, para verificar se são estatisticamente distintos em relação aos indicadores considerados nos agrupamentos. Após a comparação dos grupos, em cada um dos censos, realizou-se a confecção de mapas temáticos para representar espacialmente o comportamento dos grupos no estado do RS. Isto é feito para verificar se existem semelhanças geográficas nos grupos formados a partir dos indicadores socioeconômicos estudados. A aplicação da análise de agrupamento, do 
teste de Kruskal-Wallis e do teste de Dunn foi realizada no software R versão 3.4.4 (R Core Team, 2018), com utilização dos pacotes MVar.pt (OSSANI e CIRILLO, 2018), NbClust (CHARRAD et al., 2014), vegan (OKSANEN et al., 2018) e dunn.test (DINNO, 2017). Os grupos foram representados por mapas temáticos, utilizando o software QGis.

\section{RESULTADOS E DISCUSSÃO}

A análise descritiva dos indicadores selecionados para os anos de 1991, 2000 e 2010 encontra-se na Tabela 1.

Tabela 1 - Análise descritiva dos indicadores Esperança de Vida (EV), Mortalidade Infantil (MI), Índice de Desenvolvimento Humano (IDH), Razão de Dependência (RD), Taxa de Analfabetismo (TANA), Expectativa de Anos de Estudo (EADE), Renda per capita (RPC) e Percentual de Renda Apropriada pelos $20 \%$ mais pobres (PRAMP), nos anos de 1991,2000 e 2010

\begin{tabular}{|c|c|c|c|c|c|c|c|c|c|}
\hline Anos & & EV & $\mathrm{MI}$ & IDH & $\mathrm{RD}$ & TANA & EADE & RPC & PRAMP \\
\hline \multirow{6}{*}{1991} & Média & 68,53 & 22,72 & 0,46 & 57,76 & 13,24 & 9,69 & 322,57 & 3,55 \\
\hline & Desvio Padrão & 0,54 & 1,12 & 0,00 & 4,67 & 4,37 & 0,31 & 71,69 & 1,04 \\
\hline & Máximo & 72,29 & 39,69 & 0,66 & 82,90 & 32,25 & 12,12 & 1021,93 & 6,46 \\
\hline & Mínimo & 62,40 & 14,60 & 0,23 & 43,64 & 2,28 & 2,25 & 67,20 & 0,12 \\
\hline & Mediana & 68,63 & 22,22 & 0,47 & 57,08 & 12,80 & 10,03 & 305,05 & 3,38 \\
\hline & Coeficiente de Variação & 0,78 & 4,95 & 1,07 & 8,09 & 33,01 & 3,21 & 22,22 & 29,25 \\
\hline \multirow{6}{*}{2000} & Média & 72,39 & 18,35 & 0,61 & 51,31 & 9,21 & 10,30 & 482,07 & 3,77 \\
\hline & Desvio Padrão & 0,33 & 0,57 & 0,01 & 1,36 & 4,30 & 0,51 & 73,27 & 1,10 \\
\hline & Máximo & 76,93 & 30,50 & 0,74 & 81,38 & 24,32 & 13,02 & 1399,50 & 8,47 \\
\hline & Mínimo & 66,59 & 11,20 & 0,44 & 40,71 & 1,60 & 5,72 & 150,04 & 0,30 \\
\hline & Mediana & 72,39 & 18,15 & 0,61 & 50,92 & 8,54 & 10,40 & 464,57 & 3,59 \\
\hline & Coeficiente de Variação & 0,46 & 3,08 & 2,45 & 2,66 & 46,70 & 4,94 & 15,20 & 29,23 \\
\hline \multirow{6}{*}{2010} & Média & 75,42 & 12,42 & 0,71 & 45,09 & 6,74 & 10,24 & 742,83 & 4,51 \\
\hline & Desvio Padrão & 0,02 & 0,07 & 0,03 & 0,98 & 2,18 & 0,45 & 116,67 & 1,34 \\
\hline & Máximo & 78,38 & 19,00 & 0,81 & 69,83 & 20,11 & 12,72 & 1758,27 & 9,26 \\
\hline & Mínimo & 70,96 & 8,49 & 0,59 & 33,53 & 0,95 & 7,95 & 336,44 & 0,66 \\
\hline & Mediana & 75,52 & 12,28 & 0,72 & 45,35 & 6,41 & 10,28 & 714,13 & 4,30 \\
\hline & Coeficiente de Variação & 0,03 & 0,57 & 4,76 & 2,18 & 32,40 & 4,42 & 15,71 & 29,82 \\
\hline
\end{tabular}

Conforme dados da Tabela 1, em média todos os municípios, no decorrer dos censos tanto para a esperança de vida, como para o IDH, renda per capita e percentual de renda apropriada pelos $20 \%$ mais pobres há um crescimento considerável. Já para a mortalidade infantil, a razão de dependência e a taxa de analfabetismo ocorre uma redução para esses indicadores. Entretanto, a expectativa de anos de estudo apresenta 
inicialmente um crescimento e posteriormente uma leve redução na média dos seus valores.

Na Tabela 2 é exibida a mediana de cada indicador dentro dos agrupamentos gerados em cada censo analisado. Em Silva et al. (2019) foram apresentados a média e o rankeamento dos indicadores em cada grupo gerado. A composição dos grupos encontra-se no Apêndice.

Tabela 2 - Comparação de grupos para os indicadores Esperança de Vida (EV), Mortalidade Infantil (MI), Índice de Desenvolvimento Humano (IDH), Razão de Dependência (RD), Taxa de Analfabetismo (TANA), Expectativa de Anos de Estudo (EADE), Renda per capita (RPC) e Percentual de Renda Apropriada pelos 20\% mais pobres (PRAMP), nos anos de 1991, 2000 e 2010

\begin{tabular}{|c|c|c|c|c|c|c|c|c|c|}
\hline Ano & Grupo $\$$ & EV & $\mathrm{Ml}$ & IDH & $\mathrm{RD}$ & TANA & EADE & RPC & PRAMP \\
\hline \multirow{5}{*}{1991} & Grupo A1 & $68,02 \quad$ (a) & $23,63(a)$ & $0,44(a)$ & $58,58(a)$ & $14,74(a)$ & $9,91 \quad$ (a) & 262,27 (a) 3,15 & (a) \\
\hline & Grupo A2 & 69,87 (b) & $19,43(b)$ & $0,53(b)$ & 53,71 (b) & $8,15 \quad(b)$ & 10,37 (b) & 444,93 (b) 4,32 & (b) \\
\hline & Grupo A3 & $64,18 \quad$ (c) & 34,02 (c) & 0,33 (c) & 71,67 (c) & 27,38 (c) & $8,39 \quad$ (c) & 150,15 (c) 3,30 & (a) \\
\hline & Grupo A4 & $69,04 \quad(\#)$ & $33,34(\#)$ & $0,54(\#)$ & $53,65(\#)$ & 12,02 & 10,33 & 577,41 (\#) 1,33 & (\#) \\
\hline & Grupo A5 & $69,87 \quad(\#)$ & 21,12 (\#) & 0,66 (\#) & 50,48 (\#) & 5,23 & 10,53 & 1021,93 (\#) 2,65 & (\#) \\
\hline \multirow{4}{*}{2000} & Grupo B1 & $72,56 \quad$ (a) & $17,80(a)$ & $0,63(a)$ & 49,91 (a) & $7,36 \quad$ (a) & 10,48 & 502,85 (a) 3,85 & (a) \\
\hline & Grupo B2 & 71,11 (b) & $20,50(b)$ & $0,55(b)$ & $54,82(b)$ & 14,56 (b) & $9,87 \quad$ (b) & 324,42 (b) 2,89 & (b) \\
\hline & Grupo B3 & $74,56 \quad$ (c) & 14,90 (c) & 0,70 (c) & 44,46 (c) & 4,39 (c) & 10,78 & 868,38 (c) 4,36 & (a) \\
\hline & Grupo B4 & $73,65 \quad(\#)$ & 16,04 (\#) & 0,74 (\#) & $45,56(\#)$ & 3,45 & 10,45 & 1399,50 (\#) 2,14 & (\#) \\
\hline \multirow{4}{*}{2010} & Grupo C1 & 75,84 (a) & $12,00(a)$ & $0,73(a)$ & $43,88(a)$ & 5,19 (a) & 10,34 (a) & 777,41 (a) 4,68 & (a) \\
\hline & Grupo C2 & $76,00 \quad$ (a) & $11,70(a)$ & 0,77 (b) & $43,28(a)$ & $4,16 \quad(a)$ & $10,34(a b)$ & 1408,71 (b) 3,50 & (b) \\
\hline & Grupo C3 & 74,11 (b) & $14,00(b)$ & 0,66 (c) & 48,95 (b) & 10,71 (b) & 10,13 (b) & 531,38 (c) 3,33 & (b) \\
\hline & Grupo C4 & $74,01 \quad(b)$ & $14,15(b)$ & $0,64(c)$ & $52,53(b)$ & $17,41(b)$ & $9,77 \quad$ (c) & 463,28 (c) 2,38 & (b) \\
\hline
\end{tabular}

Em 1991 os 496 municípios ficaram divididos em cinco agrupamentos. De acordo com Silva et al. (2019), o grupo A1 foi o de maior tamanho, com 335 municípios (67,50\% dos municípios do estado).

No ano 2000 o número de grupos passa a ser 4, onde o grupo B1 foi o maior, com 346 municípios (69,76\% do total das cidades do estado do RS) (SILVA et al., 2019).

No censo de 2010 o número de grupos permaneceu o mesmo que em 2000, no entanto o número de municípios referente a cada agrupamento mudou. O grupo C1 passou a ser composto por 396 municípios, representando 78,84\% do estado (SILVA et al., 2019). 
Com base na Tabela 2 verifica-se que, para todos os indicadores, em todos os censos, ocorrem diferenças significativas para pelo menos dois grupos pelo teste de Kruskal-Wallis $(p<0,05)$.

Em relação ao censo de 1991, todos os grupos diferem entre si para os indicadores Esperança de Vida (EV), Mortalidade Infantil (MI), Índice de Desenvolvimento Humano (IDH), Razão de Dependência (RD), Taxa de Analfabetismo (TANA), Expectativa de Anos de Estudo (EADE) e Renda per capita (RPC) pelo teste de Dunn $(p<0,05)$. Já para o indicador Percentual de Renda Apropriada pelos $20 \%$ mais pobres (PRAMP) apenas o grupo A2 difere dos demais.

No censo de 2000, também ocorrem diferenças entre os grupos para os indicadores Esperança de Vida (EV), Mortalidade Infantil (MI), Índice de Desenvolvimento Humano (IDH), Razão de Dependência (RD), Taxa de Analfabetismo (TANA), Expectativa de Anos de Estudo (EADE) e Renda per capita (RPC) pelo teste de Dunn $(p<0,05)$. Já para o indicador Percentual de Renda Apropriada pelos $20 \%$ mais pobres (PRAMP) apenas o grupo B2 difere dos demais.

Assim, percebe-se uma semelhança no comportamento dos grupos, indicando que, com exceção do indicador PRAMP, todos diferem entre si. Deste modo, para os censos de 1991 e 2000, os grupos parecem bem definidos e com características estatisticamente distintas.

Em 2010, para os indicadores Esperança de Vida (EV), Mortalidade Infantil (MI), Razão de Dependência (RD), Taxa de Analfabetismo (TANA) os grupos C1 e C2 não diferiram entre si, e os grupos C3 e C4 também não, pelo teste de Dunn $(p<0,05)$. Ainda, não existe diferença ao considerar os indicadores IDH e RPC nos grupos C3 e C4. Para o indicador EADE os grupos C1 e C2 não diferiram entre si, ocorrendo o mesmo com os grupos C2 e C3. Para o indicador PRAMP apenas o grupo C1 diferiu dos demais.

Percebe-se que no censo de 2010, os grupos não apresentaram tanta distinção entre si, pois em vários indicadores, alguns grupos tiveram comportamentos semelhantes. Numa visão socioeconômica, isto revela que neste censo, os municípios estão mais semelhantes entre si, mostrando que as desigualdades socioeconômicas diminuíram. Isto também permite verificar que houve evolução ao longo dos censos, 
mostrando que as desigualdades socioeconômicas estão diminuindo também ao longo do tempo, já que em 1991 os grupos eram mais distintos entre si do que em 2010.

O relatório de Situação Social nos Estados - Rio Grande do Sul (IPEA, 2012) corrobora com os resultados aqui encontrados, mostrando um crescimento dos indicadores Esperança de Vida, Renda per capita e um decréscimo na Mortalidade Infantil e Taxa de Analfabetismo. Enfatiza ainda que a proporção de pessoas que vivia em pobreza extrema, diminuiu no período compreendido entre 2001 e 2008.

Em um estudo sobra e o Atlas Socioeconômico do RS, Cargnin et al. (2014) constataram que existem algumas regiões com diferenças significativas no Rio Grande do Sul quanto a concentração da população e desenvolvimento mas ponderam que podem ser observados núcleos de desenvolvimento pelo território gaúcho, como é o caso das regiões de Pelotas e Rio Grande, Santa Maria, Ijuí-Panambi-Santa RosaHorizontina, Erechim, dentre outros.

O grupo A3 de 1991 tem a mediana dos indicadores semelhante com o grupo B2 de 2000 e o grupo C4 de 2010, por possuírem os maiores valores para a mortalidade infantil e também para a razão de dependência, além dos menores valores para esperança de vida, pior IDH, menor expectativa de anos de estudo e mais baixa renda per capita. Esses três grupos são característicos por possuírem os piores valores dos indicadores analisados, sendo assim eles podem ser denominados como os de pior contexto socioeconômico.

A Figura 1 apresenta os mapas temáticos para os três anos analisados, onde é possível observar que os grupos não dependem da posição geográfica dos seus componentes. Ou seja, não necessariamente municípios que estejam próximos geograficamente estarão no mesmo grupo.

Até é possível identificar alguns aglomerados de vizinhança, mas que não caracteriza que o grupo esteja formando um agrupamento espacial especifico. Pois o que realmente interfere na formação dos grupos são as características socioeconômicas dos municípios. 
Figura 1 - Mapas dos agrupamentos gerados nos censos de 1991, 2000 e 2010 no Rio Grande do Sul

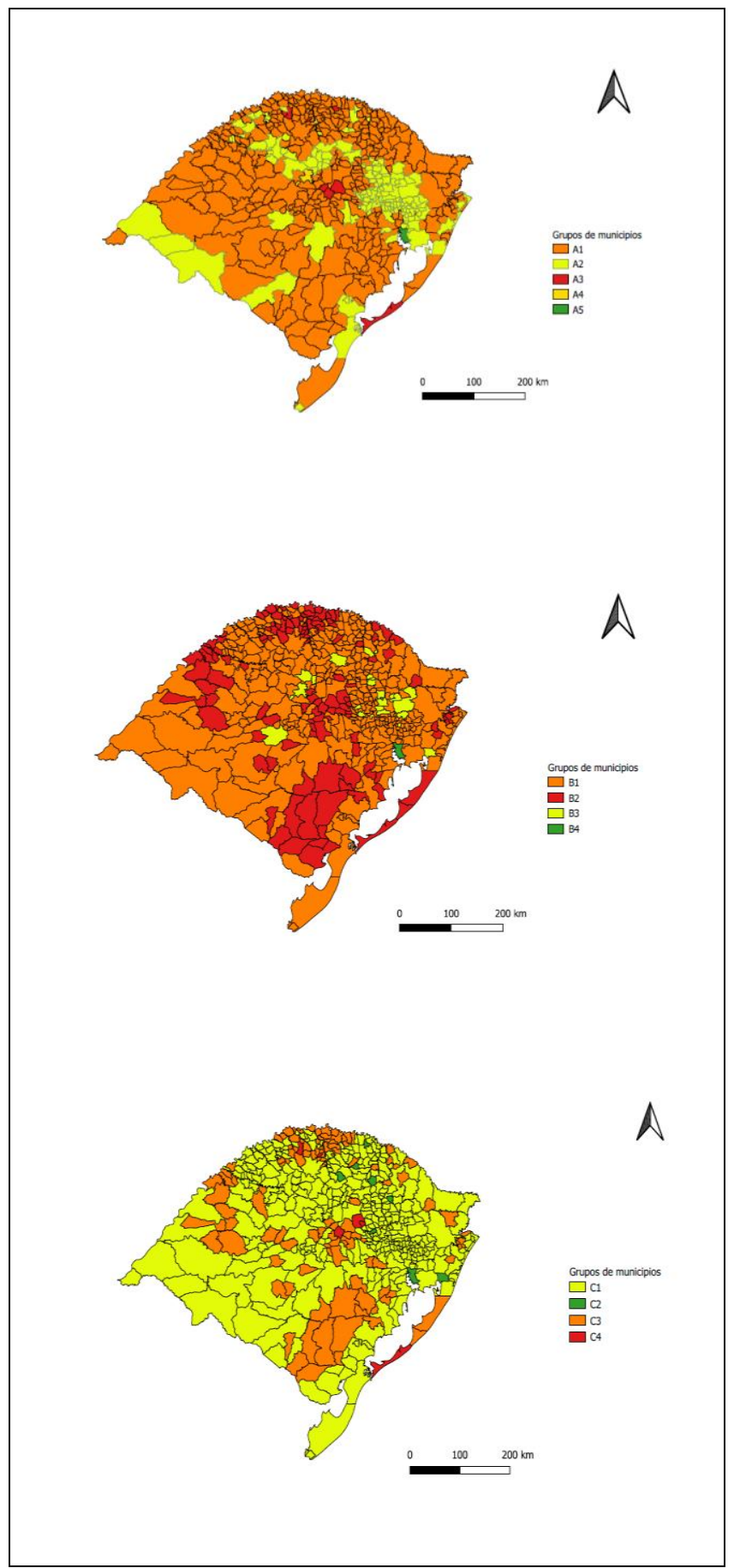

Em todos os anos comparados, os municípios de Lagoão (noroeste), Lajeado do Bugre (noroeste), Redentora (noroeste) e São José do Norte (sudeste) se mantém sempre no grupo de pior desenvolvimento socioespacial, e em 1991 e 2000 os munícipios de Barros Casal, Benjamin Constant do Sul, Braga e Tunas também compõem este agrupamento.

O grupo A5 de 1991 tem as características semelhantes com o grupo B4 de 2000 e o grupo C2 de 2010, por serem os agrupamentos que possuem o maior valor para o 
IDH, bem como para a renda per capita, e por outro lado os menores valores para razão de dependência e para a taxa de analfabetismo, podendo então caracterizar esses agrupamentos por serem aqueles que possuem os melhores valores para as variáveis analisadas, assim sendo o que possui o melhor contexto socioeconômico.

O que existe de igual nos agrupamentos que representam o melhor contexto socioespacial é que nos três anos analisados o município de Porto Alegre (metropolitana), pertence a esse agrupamento. Somente no ano de 2010 ele é composto por outros municípios além da capital, sendo eles: Água Santa (noroeste), Capivari do Sul (metropolitana), Coqueiros do Sul (noroeste), Guabiju (nordeste), Imbé (metropolitana), Ipiranga do Sul (noroeste), Nova Bréscia (centro oriental) e Três Arroios (noroeste). Ao comparar os indicadores dos municípios que começaram a compor o agrupamento em 2010, em relação aos três anos de comparação é perceptível o melhoramento dos indicadores,

O grupo A1 de 1991, o B1 de 2000 e o grupo C3 de 2010 são aqueles que possuem o segundo maior valor para a razão de dependência, para a taxa de analfabetismo, e para o percentual de renda apropriada pelos $20 \%$ mais pobres, assim como possuem o segundo valor mais baixo para esperança de vida e para a renda per capita. Devido a isso, podemos caracterizar esses grupos por serem os municípios com o segundo pior nível socioeconômico.

Assim os municípios que se mantêm no agrupamento de segundo pior nível socioeconômico nos três censos são: Boa Vista das Missões (noroeste), Chuvisca (metropolitana), General Câmera (metropolitana), Iraí (noroeste), Jaquirama (nordeste), Jari (centro ocidental), Manoel Viana (sudoeste), Pântano Grande (centro oriental), Pinhal Grande (centro ocidental), São Miguel das Missões (noroeste), Sertão Santana (metropolitana), Sinimbu (centro oriental) e Tupanci do Sul (noroeste).

Já os grupos A2 e A4 de 1991 se assemelham com o B3 de 2000 e com o C1 de 2010, sendo eles caracterizados por possuírem baixos valores para mortalidade infantil, razão de dependência e taxa de analfabetismo, e valores altos para renda per capita e esperança de vida, assim podendo ser descrito pelos municípios com o 
segundo melhor cenário socioeconômico. Percebe-se que em 2010 existem mais municípios que melhoraram os seus indicadores.

Dentre os munícipios analisados, os que podem ser denominados como os com segundo melhor cenário socioeconômico nos três anos são: Arroio do Meio (centro oriental), Bento Gonçalves (nordeste), Carlos Barbosa (nordeste), Caxias do Sul (nordeste), Flores da Cunha (nordeste), Fortaleza dos Vales (noroeste), Garibaldi (nordeste), Ibirubá (noroeste), Ivoti (metropolitana), Nova Araçá (nordeste), Nova Bassano (nordeste), Nova Pádua (nordeste), Nova Petrópolis (metropolitana), Paraí (nordeste), Passo Fundo (noroeste), Santa Maria (centro ocidental), Selbach (noroeste), Veranópolis (nordeste) e Westfalia (centro oriental).

Os resultados obtidos neste estudo corroboram com os estudos de Concha (2013), Trennepohl e Paiva (2012) e Schneider e Waquil (2001), que demonstram que o Rio Grande do Sul possui uma grande heterogeneidade em seus municípios, decorrente de questões sociais. Contudo, argumenta-se que tais heterogeneidades tendem a diminuir ao decorrer do tempo, pelo menos em alguns dos indicadores considerados.

\section{CONCLUSÕES}

A partir dos grupos gerados foi possível identificar que o estado do Rio Grande do Sul apresenta uma grande heterogeneidade socioeconômica, havendo aqueles que apresentam altos níveis de desenvolvimento, bem como aqueles com baixos níveis de desenvolvimento. Desta forma, conforme a análise de agrupamento, no ano de 1991 os municípios gaúchos se distribuíram em cinco grupos distintos. Em 2000 e 2010 essa distribuição ocorreu em quatro grupos.

No ano de 2010 oito municípios tiveram um bom desempenho nos indicadores e se agruparam com a capital do estado, que é a cidade com melhor desenvolvimento nos três anos que são analisados. Houve um crescimento de oito para 127 municípios nos grupos que ficaram caracterizados por possuírem o pior perfil socioespacial de 1991 para 2000. Entretanto, no ano de 2010 o número caiu para apenas seis municípios. Salienta-se ainda que em sua grande maioria, há diferenças estatísticas 
significativas entre os grupos de municípios gerados no que tange aos indicadores analisados.

Já os grupos que possuem o segundo pior comportamento socioeconômico tiveram um aumento de municípios de 1991 para 2000, passando de 335 para 346. Porém, no ano de 2010 o grupo que possui essas características, diminuiu para menos que a metade, com um total de 112 municípios. Contudo os agrupamentos com o segundo melhor perfil socioeconômico tiveram uma queda de 1991 para 2000, entretanto no ano de 2010 o número de municípios pertencentes a esse agrupamento foi duas vezes maior que no primeiro ano analisado.

Assim, a partir dos agrupamentos gerados é possível traçar estratégias de políticas públicas e priorizar as medidas que devem ser tomadas pelos órgãos competentes, em cada uma das regiões. 


\section{REFERÊNCIAS}

ALONSO JAF, BENETTI MD, BANDEIRA PS. Crescimento econômico da região sul do Rio Grande do Sul: causas e perspectivas. Edições FEE, 1984 [Internet]: Porto Alegre: http://cdn.fee.tche.br/digitalizacao/crescimento-economico-regiao-sul-rio-grande-dosul-causas-perspectivas/crescimento-economico-regiao-sul-rio-grande-do-sul-causasperspectivas-texto.pdf

ATLAS BRASIL; Atlas do desenvolvimento humano - Rio Grande do Sul. Disponível em: http://www.atlasbrasil.org.br/2013/pt/perfil_uf/rio-grande-do-sul. Acesso em: 10/10/2017.

CARGNIN AP, BERTÊ AMA, LEMOS BO, OLIVEIRA SB. Quinze anos de transformações na economia e sociedade gaúchas contados pelas páginas do atlas socioeconômico do RS. Boletim Geográfico do Rio Grande do Sul. 2014; 24:29-62

CASTRO LS, MIRANDA MH, LIMA JE. Indicadores sociais de desenvolvimento e a produção de soja: uma análise multivariada nos 150 maiores municípios produtores brasileiros. Revista Brasileira de Gestão e Desenvolvimento Regional. 2015;11(1):69-87.

CHARRAD M, GHAZZALI N, BOITEAU V, NIKNAFS A. NbClust: An R package for determining the relevant number of clusters in a data set. Journal of Statistical Software. 2014;61(6):1-36.

CONCHA M., WAQUIL PD, SCHNEIDER S. Tipologia dos municípios gaúchos com base nos dados dos Censos Agropecuários de 2006 e Censo demográfico de 2010. Ensaios FEE. 2013;34:938-1006.

CRISPIM DL, FERNANDES, LL, ALBUQUERQUE RLO. Aplicação de técnica estatística multivariada em indicadores de sustentabilidade nos municípios do Marajó-PA. Revista Principia. 2019; 46:145-154.

DINNO A. dunn.test: Dunn's Test of Multiple Comparisons Using Rank Sums. $R$ package version 1.3.5., 2017. Disponível em: https://CRAN.Rproject.org/package $=$ dunn. test.

IPEA. Situação Social nos Estados - Rio Grande do Sul. 2012. Disponível em: http://www.ipea.gov.br/portal/images/stories/PDFs/situacao_social/120131_relatorio_ situacaosocial_rs.pdf.

JANNUZZI PM. Indicadores Sociais no Brasil. 5 st ed. Campinas, São Paulo: Editora Alínea; 2012. 
LEITE GB, BRIGATTE H, AGUILAR EB. Analise Multivariada de Indicadores Socioeconomicos dos Países do G-20. Revista de Economia Mackenzie. 2009;7(1):125147.

OKSANEN J, BLANCHET FG, FRIENDLY M, KINDT R, LEGENFRE P. et al. Vegan: Community Ecology Package. R package version 2.5-2, 2018.https://CRAN.Rproject.org/package=vegan

OSSANI PC, CIRILLO MA. MVar. Pt: Analise Multivariada. R package version 2.0.1, 2018.

PONTES RP, LIMA JE. Índice de desenvolvimento econômico das famílias no Rio Grande do Sul: Uma abordagem por intermédio da análise multivariada. Estudo \& Debate. 2018;25(1):237-260.

R Core Team (2018). R: A language and environment for statistical computing. R Foundation for Statistical Computing, Vienna, Áustria. Disponível em: https://www.Rproject.org/.

SCHNEIDER S, WAQUIL PD. Caracterização socioeconômica dos munícipios gaúchos e desigualdades regionais. Revista de Economia e Sociologia Rural. 2001;39(3):117142.

SILVA MMD, SILVA AM, SEIDEL EJ, MATEUS ALSS, ANSUJ AP. Agrupamento Socioeconômico de Municípios do Rio Grande do Sul. In: Anais da 64 Rbras e 18 SEAGRO. 2019; Cuiaba, Brasil.

TRENNEPOHL D, PAIVA CAN. Contribuição potencial de atividades agropecuárias selecionadas para o desenvolvimento regional do Noroeste gaúcho. Revista Brasileira de Gestão e Desenvolvimento. 2012;8(1):232-268. 
Apêndice - Lista dos municípios e seus respectivos grupos nos anos de 1991, 2000 e 2010

\begin{tabular}{|c|c|c|c|}
\hline Municípios & 1991 & 2000 & 2010 \\
\hline Aceguá (RS) & A1 & B1 & $\mathrm{C} 1$ \\
\hline Água Santa (RS) & $\mathrm{A} 1$ & B1 & $\mathrm{C} 2$ \\
\hline Agudo (RS) & $\mathrm{A} 1$ & B1 & $\mathrm{C} 1$ \\
\hline Ajuricaba (RS) & A1 & B1 & C1 \\
\hline Alecrim (RS) & $\mathrm{A} 1$ & B1 & C1 \\
\hline Alegrete (RS) & $\mathrm{A} 1$ & B1 & C1 \\
\hline Alegria (RS) & A1 & B2 & C3 \\
\hline Almirante Tamandaré do Sul (RS) & $\mathrm{A} 1$ & B1 & C1 \\
\hline Alpestre (RS) & $\mathrm{A} 1$ & B2 & C3 \\
\hline Alto Alegre (RS) & $\mathrm{A} 1$ & B1 & $\mathrm{C} 1$ \\
\hline Alto Feliz (RS) & $\mathrm{A} 2$ & B1 & C1 \\
\hline Alvorada (RS) & $\mathrm{A} 2$ & B1 & $\mathrm{C} 1$ \\
\hline Amaral Ferrador (RS) & $\mathrm{A} 1$ & B2 & $\mathrm{C} 3$ \\
\hline Ametista do Sul (RS) & $\mathrm{A} 1$ & B2 & $\mathrm{C} 3$ \\
\hline André da Rocha (RS) & $\mathrm{A} 1$ & B1 & $\mathrm{C} 1$ \\
\hline Anta Gorda (RS) & $\mathrm{A} 1$ & B1 & $\mathrm{C} 1$ \\
\hline Antônio Prado (RS) & $\mathrm{A} 2$ & B1 & $\mathrm{C} 1$ \\
\hline Arambaré (RS) & $\mathrm{A} 1$ & B2 & $\mathrm{C} 1$ \\
\hline Araricá (RS) & $\mathrm{A} 1$ & B1 & $\mathrm{C} 1$ \\
\hline Aratiba (RS) & $\mathrm{A} 1$ & B1 & $\mathrm{C} 1$ \\
\hline Arroio do Meio (RS) & $\mathrm{A} 2$ & B3 & $\mathrm{C} 1$ \\
\hline Arroio do Padre (RS) & $\mathrm{A} 1$ & B1 & $\mathrm{C} 1$ \\
\hline Arroio do Sal (RS) & $\mathrm{A} 2$ & B1 & $\mathrm{C} 1$ \\
\hline Arroio dos Ratos (RS) & $\mathrm{A} 1$ & B1 & $\mathrm{C} 1$ \\
\hline Arroio do Tigre (RS) & $\mathrm{A} 1$ & B1 & $\mathrm{C} 1$ \\
\hline Arroio Grande (RS) & A1 & B2 & $\mathrm{C} 1$ \\
\hline Arvorezinha (RS) & $\mathrm{A} 1$ & B1 & $\mathrm{C} 1$ \\
\hline Augusto Pestana (RS) & $\mathrm{A} 2$ & B1 & $\mathrm{C} 1$ \\
\hline Áurea (RS) & $\mathrm{A} 1$ & B1 & $\mathrm{C} 1$ \\
\hline Bagé (RS) & $\mathrm{A} 2$ & B1 & $\mathrm{C} 1$ \\
\hline Balneário Pinhal (RS) & $\mathrm{A} 1$ & B1 & $\mathrm{C} 1$ \\
\hline Barão de Cotegipe (RS) & $\mathrm{A} 1$ & B1 & $\mathrm{C} 1$ \\
\hline Barão do Triunfo (RS) & $\mathrm{A} 1$ & B2 & $\mathrm{C} 3$ \\
\hline Barão (RS) & $\mathrm{A} 2$ & B1 & $\mathrm{C} 1$ \\
\hline Barracão (RS) & $\mathrm{A} 1$ & B2 & $\mathrm{C} 1$ \\
\hline Barra do Guarita (RS) & $\mathrm{A} 1$ & B2 & $\mathrm{C} 3$ \\
\hline Barra do Quaraí (RS) & $\mathrm{A} 1$ & B1 & $\mathrm{C} 1$ \\
\hline Barra do Ribeiro (RS) & $\mathrm{A} 1$ & B1 & $\mathrm{C} 1$ \\
\hline Barra do Rio Azul (RS) & $\mathrm{A} 1$ & B1 & $\mathrm{C} 1$ \\
\hline Barra Funda (RS) & $\mathrm{A} 1$ & B1 & $\mathrm{C} 1$ \\
\hline Barros Cassal (RS) & A3 & B2 & $\mathrm{C} 3$ \\
\hline Benjamin Constant do Sul (RS) & A3 & B2 & C3 \\
\hline Bento Gonçalves (RS) & $\mathrm{A} 2$ & B3 & $\mathrm{C} 1$ \\
\hline Boa Vista das Missões (RS) & $\mathrm{A} 1$ & B1 & $\mathrm{C} 3$ \\
\hline Boa Vista do Buricá (RS) & $\mathrm{A} 2$ & B1 & $\mathrm{C} 1$ \\
\hline Boa Vista do Cadeado (RS) & $\mathrm{A} 1$ & B1 & $\mathrm{C} 1$ \\
\hline Boa Vista do Incra (RS) & $\mathrm{A} 1$ & B1 & $\mathrm{C} 1$ \\
\hline Boa Vista do Sul (RS) & $\mathrm{A} 2$ & $\mathrm{~B} 1$ & C1 \\
\hline
\end{tabular}




\begin{tabular}{|c|c|c|c|}
\hline Bom Jesus (RS) & A1 & B1 & $\mathrm{C} 1$ \\
\hline Bom Princípio (RS) & $\mathrm{A} 2$ & B1 & C1 \\
\hline Bom Progresso (RS) & $\mathrm{A} 1$ & B2 & $\mathrm{C} 3$ \\
\hline Bom Retiro do Sul (RS) & $\mathrm{A} 2$ & B1 & $\mathrm{C} 1$ \\
\hline Boqueirão do Leão (RS) & $\mathrm{A} 1$ & B2 & $\mathrm{C} 3$ \\
\hline Bossoroca (RS) & $\mathrm{A} 1$ & B2 & C1 \\
\hline Bozano (RS) & $\mathrm{A} 2$ & B1 & C1 \\
\hline Braga (RS) & A3 & B2 & C3 \\
\hline Brochier (RS) & $\mathrm{A} 2$ & B1 & C1 \\
\hline Butiá (RS) & $\mathrm{A} 1$ & B1 & C1 \\
\hline Caçapava do Sul (RS) & $\mathrm{A} 1$ & B1 & C1 \\
\hline Cacequi (RS) & $\mathrm{A} 1$ & B1 & C1 \\
\hline Cachoeira do Sul (RS) & $\mathrm{A} 2$ & B1 & C1 \\
\hline Cachoeirinha (RS) & $\mathrm{A} 2$ & B1 & C1 \\
\hline Cacique Doble (RS) & $\mathrm{A} 1$ & B2 & C3 \\
\hline Caibaté (RS) & $\mathrm{A} 1$ & B1 & $\mathrm{C} 1$ \\
\hline Caiçara (RS) & $\mathrm{A} 1$ & B1 & $\mathrm{C} 1$ \\
\hline Camaquã (RS) & A1 & B1 & C1 \\
\hline Camargo (RS) & A1 & B1 & C1 \\
\hline Cambará do Sul (RS) & $\mathrm{A} 1$ & B1 & $\mathrm{C} 1$ \\
\hline Campestre da Serra (RS) & $\mathrm{A} 1$ & B1 & C1 \\
\hline Campina das Missões (RS) & $\mathrm{A} 1$ & B1 & C1 \\
\hline Campinas do Sul (RS) & $\mathrm{A} 1$ & B1 & C1 \\
\hline Campo Bom (RS) & $\mathrm{A} 2$ & B1 & C1 \\
\hline Campo Novo (RS) & $\mathrm{A} 1$ & B2 & C3 \\
\hline Campos Borges (RS) & $\mathrm{A} 1$ & B2 & C1 \\
\hline Candelária (RS) & $\mathrm{A} 1$ & B2 & $\mathrm{C} 3$ \\
\hline Cândido Godói (RS) & $\mathrm{A} 1$ & B1 & $\mathrm{C} 1$ \\
\hline Candiota (RS) & $\mathrm{A} 1$ & B1 & C1 \\
\hline Canela (RS) & $\mathrm{A} 2$ & B1 & C1 \\
\hline Canguçu (RS) & $\mathrm{A} 1$ & B2 & C3 \\
\hline Canoas (RS) & A2 & B1 & C1 \\
\hline Canudos do Vale (RS) & A1 & B2 & $\mathrm{C} 1$ \\
\hline Capão Bonito do Sul (RS) & $\mathrm{A} 1$ & B2 & C3 \\
\hline Capão da Canoa (RS) & $\mathrm{A} 2$ & B1 & C1 \\
\hline Capão do Cipó (RS) & $\mathrm{A} 1$ & B1 & $\mathrm{C} 1$ \\
\hline Capão do Leão (RS) & $\mathrm{A} 1$ & B1 & C1 \\
\hline Capela de Santana (RS) & $\mathrm{A} 1$ & B1 & C1 \\
\hline Capitão (RS) & $\mathrm{A} 2$ & B1 & $\mathrm{C} 1$ \\
\hline Capivari do Sul (RS) & $\mathrm{A} 1$ & B3 & $\mathrm{C} 2$ \\
\hline Caraá (RS) & $\mathrm{A} 1$ & B2 & C3 \\
\hline Carazinho (RS) & $\mathrm{A} 2$ & B1 & C1 \\
\hline Carlos Barbosa (RS) & $\mathrm{A} 2$ & B3 & C1 \\
\hline Carlos Gomes (RS) & $\mathrm{A} 2$ & B1 & $\mathrm{C} 1$ \\
\hline Casca (RS) & $\mathrm{A} 1$ & B1 & C1 \\
\hline Caseiros (RS) & $\mathrm{A} 1$ & B2 & C3 \\
\hline Catuípe (RS) & $\mathrm{A} 2$ & B1 & C1 \\
\hline Caxias do Sul (RS) & $\mathrm{A} 2$ & B3 & C1 \\
\hline Centenário (RS) & $\mathrm{A} 1$ & B1 & $\mathrm{C} 1$ \\
\hline Cerrito (RS) & $\mathrm{A} 1$ & B2 & C3 \\
\hline Cerro Branco (RS) & $\mathrm{A} 1$ & B2 & C3 \\
\hline Cerro Grande do Sul (RS) & $\mathrm{A} 1$ & $\mathrm{~B} 2$ & $\mathrm{C} 3$ \\
\hline
\end{tabular}




\begin{tabular}{|c|c|c|c|}
\hline Cerro Grande (RS) & A1 & B2 & $\mathrm{C} 3$ \\
\hline Cerro Largo (RS) & $\mathrm{A} 1$ & B1 & $\mathrm{C} 1$ \\
\hline Chapada (RS) & $\mathrm{A} 1$ & B1 & C1 \\
\hline Charqueadas (RS) & $\mathrm{A} 2$ & B1 & $\mathrm{C} 1$ \\
\hline Charrua (RS) & $\mathrm{A} 1$ & B2 & $\mathrm{C} 3$ \\
\hline Chiapetta (RS) & $\mathrm{A} 1$ & B1 & C1 \\
\hline Chuí (RS) & $\mathrm{A} 2$ & B1 & $\mathrm{C} 1$ \\
\hline Chuvisca (RS) & $\mathrm{A} 1$ & B1 & $\mathrm{C3}$ \\
\hline Cidreira (RS) & $\mathrm{A} 1$ & B1 & $\mathrm{C} 1$ \\
\hline Ciríaco (RS) & $\mathrm{A} 1$ & B1 & $\mathrm{C} 1$ \\
\hline Colinas (RS) & $\mathrm{A} 2$ & B1 & C1 \\
\hline Colorado (RS) & $\mathrm{A} 2$ & B1 & $\mathrm{C} 1$ \\
\hline Condor (RS) & $\mathrm{A} 1$ & B1 & C1 \\
\hline Constantina (RS) & $\mathrm{A} 1$ & B1 & $\mathrm{C} 1$ \\
\hline Coqueiro Baixo (RS) & $\mathrm{A} 1$ & B1 & $\mathrm{C} 1$ \\
\hline Coqueiros do Sul (RS) & $\mathrm{A} 1$ & B1 & $\mathrm{C} 2$ \\
\hline Coronel Barros (RS) & $\mathrm{A} 2$ & B1 & $\mathrm{C} 1$ \\
\hline Coronel Bicaco (RS) & $\mathrm{A} 1$ & B2 & $\mathrm{C3}$ \\
\hline Coronel Pilar (RS) & $\mathrm{A} 2$ & B1 & $\mathrm{C} 1$ \\
\hline Cotiporã (RS) & $\mathrm{A} 2$ & B1 & $\mathrm{C} 1$ \\
\hline Coxilha (RS) & $\mathrm{A} 1$ & B1 & $\mathrm{C} 1$ \\
\hline Crissiumal (RS) & $\mathrm{A} 1$ & B1 & $\mathrm{C} 1$ \\
\hline Cristal do Sul (RS) & $\mathrm{A} 1$ & B2 & $\mathrm{C3}$ \\
\hline Cristal (RS) & $\mathrm{A} 1$ & B2 & $\mathrm{C} 3$ \\
\hline Cruz Alta (RS) & $\mathrm{A} 2$ & B1 & $\mathrm{C} 1$ \\
\hline Cruzaltense (RS) & $\mathrm{A} 1$ & B1 & $\mathrm{C} 1$ \\
\hline Cruzeiro do Sul (RS) & $\mathrm{A} 2$ & B1 & $\mathrm{C} 1$ \\
\hline David Canabarro (RS) & $\mathrm{A} 1$ & B1 & $\mathrm{C} 1$ \\
\hline Derrubadas (RS) & $\mathrm{A} 1$ & B2 & $\mathrm{C} 3$ \\
\hline Dezesseis de Novembro (RS) & $\mathrm{A} 1$ & B2 & $\mathrm{C} 3$ \\
\hline Dilermando de Aguiar (RS) & $\mathrm{A} 1$ & B2 & $\mathrm{C} 3$ \\
\hline Dois Irmãos das Missões (RS) & $\mathrm{A} 1$ & B2 & $\mathrm{C} 3$ \\
\hline Dois Irmãos (RS) & $\mathrm{A} 2$ & B1 & $\mathrm{C} 1$ \\
\hline Dois Lajeados (RS) & $\mathrm{A} 2$ & B1 & $\mathrm{C} 1$ \\
\hline Dom Feliciano (RS) & $\mathrm{A} 1$ & B2 & $\mathrm{C} 3$ \\
\hline Dom Pedrito (RS) & $\mathrm{A} 1$ & B1 & $\mathrm{C} 1$ \\
\hline Dom Pedro de Alcântara (RS) & $\mathrm{A} 1$ & B1 & $\mathrm{C} 1$ \\
\hline Dona Francisca (RS) & $\mathrm{A} 1$ & B1 & $\mathrm{C} 1$ \\
\hline Doutor Maurício Cardoso (RS) & $\mathrm{A} 1$ & B1 & $\mathrm{C} 1$ \\
\hline Doutor Ricardo (RS) & $\mathrm{A} 1$ & B1 & $\mathrm{C} 1$ \\
\hline Eldorado do Sul (RS) & $\mathrm{A} 1$ & B1 & $\mathrm{C} 1$ \\
\hline Encantado (RS) & $\mathrm{A} 2$ & B1 & $\mathrm{C} 1$ \\
\hline Encruzilhada do Sul (RS) & $\mathrm{A} 1$ & B2 & $\mathrm{C} 3$ \\
\hline Engenho Velho (RS) & $\mathrm{A} 1$ & B2 & $\mathrm{C} 3$ \\
\hline Entre-Ijuís (RS) & $\mathrm{A} 1$ & B1 & $\mathrm{C} 1$ \\
\hline Entre Rios do Sul (RS) & $\mathrm{A} 1$ & B1 & $\mathrm{C} 1$ \\
\hline Erebango (RS) & $\mathrm{A} 1$ & B1 & $\mathrm{C} 1$ \\
\hline Erechim (RS) & $\mathrm{A} 2$ & B1 & $\mathrm{C} 1$ \\
\hline Ernestina (RS) & $\mathrm{A} 1$ & B1 & $\mathrm{C} 1$ \\
\hline Erval Grande (RS) & $\mathrm{A} 1$ & B2 & $\mathrm{C} 3$ \\
\hline Erval Seco (RS) & $\mathrm{A} 1$ & $\mathrm{~B} 2$ & $\mathrm{C} 3$ \\
\hline Esmeralda (RS) & $\mathrm{A} 1$ & $\mathrm{~B} 1$ & $\mathrm{C} 1$ \\
\hline
\end{tabular}




\begin{tabular}{|c|c|c|c|}
\hline Esperança do Sul (RS) & A1 & B2 & $\mathrm{C3}$ \\
\hline Espumoso (RS) & $\mathrm{A} 1$ & B1 & C1 \\
\hline Estação (RS) & $\mathrm{A} 2$ & B1 & $\mathrm{C} 1$ \\
\hline Estância Velha (RS) & $\mathrm{A} 2$ & B1 & $\mathrm{C} 1$ \\
\hline Esteio (RS) & $\mathrm{A} 2$ & B1 & $\mathrm{C} 1$ \\
\hline Estrela (RS) & $\mathrm{A} 2$ & B1 & $\mathrm{C} 1$ \\
\hline Estrela Velha (RS) & $\mathrm{A} 1$ & B2 & $\mathrm{C} 3$ \\
\hline Eugênio de Castro (RS) & $\mathrm{A} 1$ & B1 & C1 \\
\hline Fagundes Varela (RS) & $\mathrm{A} 2$ & B1 & $\mathrm{C} 1$ \\
\hline Farroupilha (RS) & $\mathrm{A} 2$ & B1 & $\mathrm{C} 1$ \\
\hline Faxinal do Soturno (RS) & $\mathrm{A} 1$ & B1 & $\mathrm{C} 1$ \\
\hline Faxinalzinho (RS) & $\mathrm{A} 1$ & B2 & $\mathrm{C} 3$ \\
\hline Fazenda Vilanova (RS) & $\mathrm{A} 1$ & B1 & $\mathrm{C} 1$ \\
\hline Feliz (RS) & $\mathrm{A} 2$ & B1 & $\mathrm{C} 1$ \\
\hline Flores da Cunha (RS) & $\mathrm{A} 2$ & B3 & $\mathrm{C} 1$ \\
\hline Floriano Peixoto (RS) & $\mathrm{A} 1$ & B1 & $\mathrm{C} 1$ \\
\hline Fontoura Xavier (RS) & $\mathrm{A} 1$ & B2 & $\mathrm{C} 4$ \\
\hline Formigueiro (RS) & $\mathrm{A} 1$ & B2 & $\mathrm{C} 3$ \\
\hline Forquetinha (RS) & $\mathrm{A} 1$ & B1 & $\mathrm{C} 1$ \\
\hline Fortaleza dos Valos (RS) & A4 & B3 & $\mathrm{C} 1$ \\
\hline Frederico Westphalen (RS) & $\mathrm{A} 1$ & B1 & $\mathrm{C} 1$ \\
\hline Garibaldi (RS) & $\mathrm{A} 2$ & B3 & $\mathrm{C} 1$ \\
\hline Garruchos (RS) & $\mathrm{A} 1$ & B2 & $\mathrm{C} 3$ \\
\hline Gaurama (RS) & $\mathrm{A} 1$ & B1 & $\mathrm{C} 1$ \\
\hline General Câmara (RS) & $\mathrm{A} 1$ & B1 & $\mathrm{C3}$ \\
\hline Gentil (RS) & $\mathrm{A} 1$ & B1 & $\mathrm{C} 1$ \\
\hline Getúlio Vargas (RS) & $\mathrm{A} 1$ & B1 & $\mathrm{C} 1$ \\
\hline Giruá (RS) & $\mathrm{A} 1$ & B1 & $\mathrm{C} 1$ \\
\hline Glorinha (RS) & $\mathrm{A} 1$ & B1 & $\mathrm{C} 1$ \\
\hline Gramado dos Loureiros (RS) & $\mathrm{A} 1$ & B2 & $\mathrm{C} 3$ \\
\hline Gramado (RS) & $\mathrm{A} 2$ & B1 & $\mathrm{C} 1$ \\
\hline Gramado Xavier (RS) & $\mathrm{A} 1$ & B2 & $\mathrm{C} 3$ \\
\hline Gravataí (RS) & $\mathrm{A} 2$ & B1 & $\mathrm{C} 1$ \\
\hline Guabiju (RS) & $\mathrm{A} 2$ & B1 & $\mathrm{C} 2$ \\
\hline Guaíba (RS) & $\mathrm{A} 2$ & B1 & $\mathrm{C} 1$ \\
\hline Guaporé (RS) & $\mathrm{A} 2$ & B1 & $\mathrm{C} 1$ \\
\hline Guarani das Missões (RS) & $\mathrm{A} 1$ & B1 & $\mathrm{C} 1$ \\
\hline Harmonia (RS) & $\mathrm{A} 2$ & B1 & $\mathrm{C} 1$ \\
\hline Herval (RS) & $\mathrm{A} 1$ & B2 & C3 \\
\hline Herveiras (RS) & $\mathrm{A} 1$ & B2 & $\mathrm{C} 3$ \\
\hline Horizontina (RS) & $\mathrm{A} 2$ & B1 & $\mathrm{C} 1$ \\
\hline Hulha Negra (RS) & $\mathrm{A} 1$ & B2 & $\mathrm{C} 3$ \\
\hline Humaitá (RS) & $\mathrm{A} 1$ & B1 & $\mathrm{C} 1$ \\
\hline Ibarama (RS) & $\mathrm{A} 1$ & B1 & $\mathrm{C} 1$ \\
\hline Ibiaçá (RS) & $\mathrm{A} 1$ & B3 & $\mathrm{C} 1$ \\
\hline Ibiraiaras (RS) & $\mathrm{A} 1$ & B1 & $\mathrm{C} 1$ \\
\hline Ibirapuitã (RS) & $\mathrm{A} 1$ & B2 & $\mathrm{C} 3$ \\
\hline Ibirubá (RS) & $\mathrm{A} 2$ & B3 & $\mathrm{C} 1$ \\
\hline Igrejinha (RS) & $\mathrm{A} 2$ & B1 & $\mathrm{C} 1$ \\
\hline ljuí (RS) & $\mathrm{A} 2$ & B1 & $\mathrm{C} 1$ \\
\hline Ilópolis (RS) & $\mathrm{A} 1$ & B1 & $\mathrm{C} 1$ \\
\hline Imbé (RS) & $\mathrm{A} 2$ & $\mathrm{~B} 1$ & $\mathrm{C} 2$ \\
\hline
\end{tabular}




\begin{tabular}{|c|c|c|c|}
\hline Imigrante (RS) & $\mathrm{A} 2$ & B1 & $\mathrm{C1}$ \\
\hline Independência (RS) & $\mathrm{A} 1$ & B1 & $\mathrm{C} 1$ \\
\hline Inhacorá (RS) & $\mathrm{A} 1$ & B2 & $\mathrm{C} 3$ \\
\hline Ipê (RS) & $\mathrm{A} 2$ & B1 & $\mathrm{C} 1$ \\
\hline Ipiranga do Sul (RS) & $\mathrm{A} 2$ & B1 & $\mathrm{C} 2$ \\
\hline Iraí (RS) & $\mathrm{A} 1$ & B1 & $\mathrm{C} 3$ \\
\hline Itaara (RS) & $\mathrm{A} 1$ & B1 & $\mathrm{C} 1$ \\
\hline Itacurubi (RS) & $\mathrm{A} 1$ & $\mathrm{~B} 2$ & $\mathrm{C3}$ \\
\hline Itapuca (RS) & $\mathrm{A} 1$ & B2 & $\mathrm{C} 1$ \\
\hline Itaqui (RS) & $\mathrm{A} 1$ & B1 & $\mathrm{C} 1$ \\
\hline Itatiba do Sul (RS) & $\mathrm{A} 1$ & B2 & $\mathrm{C3}$ \\
\hline Itati (RS) & $\mathrm{A} 1$ & B2 & $\mathrm{C3}$ \\
\hline Ivorá (RS) & $\mathrm{A} 1$ & B1 & $\mathrm{C} 1$ \\
\hline Ivoti (RS) & $\mathrm{A} 2$ & B3 & $\mathrm{C} 1$ \\
\hline Jaboticaba (RS) & $\mathrm{A} 1$ & B2 & $\mathrm{C3}$ \\
\hline Jacuizinho (RS) & $\mathrm{A} 1$ & B2 & $\mathrm{C} 3$ \\
\hline Jacutinga (RS) & $\mathrm{A} 1$ & B1 & $\mathrm{C} 1$ \\
\hline Jaguarão (RS) & $\mathrm{A} 1$ & B1 & $\mathrm{C} 1$ \\
\hline Jaguari (RS) & $\mathrm{A} 1$ & B1 & $\mathrm{C} 1$ \\
\hline Jaquirana (RS) & $\mathrm{A} 1$ & B1 & $\mathrm{C3}$ \\
\hline Jari (RS) & $\mathrm{A} 1$ & B1 & $\mathrm{C3}$ \\
\hline Jóia (RS) & $\mathrm{A} 1$ & B1 & $\mathrm{C} 1$ \\
\hline Júlio de Castilhos (RS) & $\mathrm{A} 1$ & B1 & $\mathrm{C} 1$ \\
\hline Lagoa Bonita do Sul (RS) & $\mathrm{A} 1$ & B2 & $\mathrm{C3}$ \\
\hline Lagoa dos Três Cantos (RS) & $\mathrm{A} 2$ & B1 & $\mathrm{C} 1$ \\
\hline Lagoão (RS) & A3 & B2 & $\mathrm{C} 4$ \\
\hline Lagoa Vermelha (RS) & $\mathrm{A} 1$ & B1 & $\mathrm{C} 1$ \\
\hline Lajeado do Bugre (RS) & A3 & $\mathrm{B} 2$ & $\mathrm{C} 4$ \\
\hline Lajeado (RS) & $\mathrm{A} 2$ & B1 & $\mathrm{C} 1$ \\
\hline Lavras do Sul (RS) & $\mathrm{A} 1$ & B1 & $\mathrm{C} 1$ \\
\hline Liberato Salzano (RS) & $\mathrm{A} 1$ & B2 & $\mathrm{C3}$ \\
\hline Lindolfo Collor (RS) & $\mathrm{A} 2$ & B1 & $\mathrm{C} 1$ \\
\hline Linha Nova (RS) & $\mathrm{A} 2$ & B1 & $\mathrm{C} 1$ \\
\hline Maçambará (RS) & $\mathrm{A} 1$ & B2 & $\mathrm{C3}$ \\
\hline Machadinho (RS) & $\mathrm{A} 1$ & B2 & $\mathrm{C3}$ \\
\hline Mampituba (RS) & $\mathrm{A} 1$ & B2 & $\mathrm{C} 3$ \\
\hline Manoel Viana (RS) & $\mathrm{A} 1$ & B1 & $\mathrm{C3}$ \\
\hline Maquiné (RS) & $\mathrm{A} 1$ & B1 & $\mathrm{C} 1$ \\
\hline Maratá (RS) & $\mathrm{A} 2$ & B1 & $\mathrm{C} 1$ \\
\hline Marau (RS) & $\mathrm{A} 2$ & B1 & $\mathrm{C} 1$ \\
\hline Marcelino Ramos (RS) & $\mathrm{A} 1$ & B1 & $\mathrm{C} 1$ \\
\hline Mariana Pimentel (RS) & $\mathrm{A} 1$ & B1 & $\mathrm{C} 1$ \\
\hline Mariano Moro (RS) & $\mathrm{A} 1$ & B1 & $\mathrm{C} 1$ \\
\hline Marques de Souza (RS) & $\mathrm{A} 2$ & B1 & $\mathrm{C} 1$ \\
\hline Mata (RS) & $\mathrm{A} 1$ & B1 & $\mathrm{C} 1$ \\
\hline Mato Castelhano (RS) & $\mathrm{A} 1$ & B1 & $\mathrm{C} 1$ \\
\hline Mato Leitão (RS) & $\mathrm{A} 2$ & B1 & $\mathrm{C} 1$ \\
\hline Mato Queimado (RS) & $\mathrm{A} 1$ & B1 & $\mathrm{C} 1$ \\
\hline Maximiliano de Almeida (RS) & $\mathrm{A} 1$ & B1 & $\mathrm{C} 1$ \\
\hline Minas do Leão (RS) & $\mathrm{A} 1$ & $\mathrm{~B} 2$ & $\mathrm{C} 1$ \\
\hline Miraguaí (RS) & $\mathrm{A} 1$ & B2 & $\mathrm{C3}$ \\
\hline Montauri (RS) & $\mathrm{A} 2$ & B1 & $\mathrm{C} 1$ \\
\hline
\end{tabular}




\begin{tabular}{|c|c|c|c|}
\hline Monte Alegre dos Campos (RS) & A1 & B2 & $\mathrm{C} 3$ \\
\hline Monte Belo do Sul (RS) & $\mathrm{A} 2$ & B1 & C1 \\
\hline Montenegro (RS) & $\mathrm{A} 2$ & B1 & C1 \\
\hline Mormaço (RS) & A1 & B1 & $\mathrm{C} 1$ \\
\hline Morrinhos do Sul (RS) & $\mathrm{A} 1$ & B1 & $\mathrm{C} 1$ \\
\hline Morro Redondo (RS) & $\mathrm{A} 1$ & B1 & $\mathrm{C} 1$ \\
\hline Morro Reuter (RS) & $\mathrm{A} 2$ & B1 & $\mathrm{C} 1$ \\
\hline Mostardas (RS) & $\mathrm{A} 1$ & B2 & $\mathrm{C} 3$ \\
\hline Muçum (RS) & $\mathrm{A} 2$ & B1 & C1 \\
\hline Muitos Capões (RS) & $\mathrm{A} 1$ & B1 & $\mathrm{C} 1$ \\
\hline Muliterno (RS) & $\mathrm{A} 1$ & B1 & $\mathrm{C} 1$ \\
\hline Não-Me-Toque (RS) & $\mathrm{A} 2$ & B1 & $\mathrm{C} 1$ \\
\hline Nicolau Vergueiro (RS) & $\mathrm{A} 1$ & B1 & C1 \\
\hline Nonoai (RS) & $\mathrm{A} 1$ & B2 & $\mathrm{C} 3$ \\
\hline Nova Alvorada (RS) & $\mathrm{A} 1$ & B1 & $\mathrm{C} 1$ \\
\hline Nova Araçá (RS) & $\mathrm{A} 2$ & B3 & $\mathrm{C} 1$ \\
\hline Nova Bassano (RS) & $\mathrm{A} 2$ & B3 & $\mathrm{C} 1$ \\
\hline Nova Boa Vista (RS) & $\mathrm{A} 2$ & B1 & $\mathrm{C} 1$ \\
\hline Nova Bréscia (RS) & $\mathrm{A} 2$ & B3 & $\mathrm{C} 2$ \\
\hline Nova Candelária (RS) & $\mathrm{A} 2$ & B1 & $\mathrm{C} 1$ \\
\hline Nova Esperança do Sul (RS) & $\mathrm{A} 1$ & B1 & $\mathrm{C} 1$ \\
\hline Nova Hartz (RS) & $\mathrm{A} 2$ & B1 & $\mathrm{C} 1$ \\
\hline Nova Pádua (RS) & $\mathrm{A} 2$ & B3 & $\mathrm{C} 1$ \\
\hline Nova Palma (RS) & $\mathrm{A} 1$ & B1 & C1 \\
\hline Nova Petrópolis (RS) & $\mathrm{A} 2$ & B3 & C1 \\
\hline Nova Prata (RS) & $\mathrm{A} 2$ & B1 & $\mathrm{C} 1$ \\
\hline Nova Ramada (RS) & $\mathrm{A} 2$ & B1 & $\mathrm{C} 1$ \\
\hline Nova Roma do Sul (RS) & $\mathrm{A} 2$ & B1 & $\mathrm{C} 1$ \\
\hline Nova Santa Rita (RS) & $\mathrm{A} 1$ & B1 & $\mathrm{C} 1$ \\
\hline Novo Barreiro (RS) & $\mathrm{A} 1$ & B2 & $\mathrm{C} 3$ \\
\hline Novo Cabrais (RS) & $\mathrm{A} 1$ & B2 & $\mathrm{C} 1$ \\
\hline Novo Hamburgo (RS) & $\mathrm{A} 2$ & B1 & $\mathrm{C} 1$ \\
\hline Novo Machado (RS) & $\mathrm{A} 1$ & B1 & $\mathrm{C} 1$ \\
\hline Novo Tiradentes (RS) & $\mathrm{A} 1$ & B2 & $\mathrm{C} 3$ \\
\hline Novo Xingu (RS) & $\mathrm{A} 2$ & B1 & C1 \\
\hline Osório (RS) & $\mathrm{A} 2$ & B1 & $\mathrm{C} 1$ \\
\hline Paim Filho (RS) & $\mathrm{A} 1$ & B1 & $\mathrm{C} 1$ \\
\hline Palmares do Sul (RS) & $\mathrm{A} 2$ & B1 & $\mathrm{C} 1$ \\
\hline Palmeira das Missões (RS) & $\mathrm{A} 1$ & B1 & $\mathrm{C} 1$ \\
\hline Palmitinho (RS) & $\mathrm{A} 1$ & B1 & $\mathrm{C} 1$ \\
\hline Panambi (RS) & $\mathrm{A} 1$ & B1 & C1 \\
\hline Pantano Grande (RS) & $\mathrm{A} 1$ & B1 & $\mathrm{C} 3$ \\
\hline Paraí (RS) & $\mathrm{A} 2$ & B3 & $\mathrm{C} 1$ \\
\hline Paraíso do Sul (RS) & $\mathrm{A} 1$ & B1 & $\mathrm{C} 1$ \\
\hline Pareci Novo (RS) & $\mathrm{A} 2$ & B1 & $\mathrm{C} 1$ \\
\hline Parobé (RS) & $\mathrm{A} 2$ & B1 & C1 \\
\hline Passa Sete (RS) & $\mathrm{A} 1$ & B2 & $\mathrm{C} 3$ \\
\hline Passo do Sobrado (RS) & $\mathrm{A} 2$ & B1 & $\mathrm{C} 1$ \\
\hline Passo Fundo (RS) & $\mathrm{A} 2$ & B3 & $\mathrm{C} 1$ \\
\hline Paulo Bento (RS) & $\mathrm{A} 1$ & B1 & $\mathrm{C} 1$ \\
\hline Paverama (RS) & $\mathrm{A} 1$ & B1 & $\mathrm{C} 1$ \\
\hline Pedras Altas (RS) & $\mathrm{A} 1$ & $\mathrm{~B} 2$ & $\mathrm{C} 3$ \\
\hline
\end{tabular}




\begin{tabular}{|c|c|c|c|}
\hline Pedro Osório (RS) & A1 & B2 & $\mathrm{C} 1$ \\
\hline Pejuçara (RS) & $\mathrm{A} 2$ & B1 & $\mathrm{C} 1$ \\
\hline Pelotas (RS) & $\mathrm{A} 2$ & B1 & C1 \\
\hline Picada Café (RS) & $\mathrm{A} 2$ & B1 & $\mathrm{C} 1$ \\
\hline Pinhal da Serra (RS) & $\mathrm{A} 1$ & B2 & $\mathrm{C} 3$ \\
\hline Pinhal Grande (RS) & $\mathrm{A} 1$ & B1 & $\mathrm{C3}$ \\
\hline Pinhal (RS) & $\mathrm{A} 1$ & B1 & C1 \\
\hline Pinheirinho do Vale (RS) & $\mathrm{A} 1$ & B2 & $\mathrm{C} 3$ \\
\hline Pinheiro Machado (RS) & $\mathrm{A} 1$ & B2 & $\mathrm{C3}$ \\
\hline Pirapó (RS) & $\mathrm{A} 1$ & B2 & $\mathrm{C} 3$ \\
\hline Piratini (RS) & $\mathrm{A} 1$ & B2 & $\mathrm{C} 3$ \\
\hline Planalto (RS) & $\mathrm{A} 1$ & B2 & $\mathrm{C3}$ \\
\hline Poço das Antas (RS) & $\mathrm{A} 2$ & B1 & $\mathrm{C} 1$ \\
\hline Pontão (RS) & $\mathrm{A} 1$ & B1 & $\mathrm{C} 1$ \\
\hline Ponte Preta (RS) & $\mathrm{A} 1$ & B1 & $\mathrm{C} 1$ \\
\hline Portão (RS) & $\mathrm{A} 1$ & B1 & C1 \\
\hline Porto Alegre (RS) & A5 & B4 & $\mathrm{C} 2$ \\
\hline Porto Lucena (RS) & $\mathrm{A} 1$ & B2 & $\mathrm{C} 1$ \\
\hline Porto Mauá (RS) & $\mathrm{A} 1$ & B1 & $\mathrm{C} 1$ \\
\hline Porto Vera Cruz (RS) & $\mathrm{A} 1$ & B2 & $\mathrm{C} 1$ \\
\hline Porto Xavier (RS) & $\mathrm{A} 1$ & B2 & $\mathrm{C3}$ \\
\hline Pouso Novo (RS) & $\mathrm{A} 1$ & B2 & $\mathrm{C} 1$ \\
\hline Presidente Lucena (RS) & $\mathrm{A} 2$ & B1 & $\mathrm{C} 1$ \\
\hline Progresso (RS) & $\mathrm{A} 1$ & B2 & $\mathrm{C} 1$ \\
\hline Protásio Alves (RS) & $\mathrm{A} 1$ & B1 & $\mathrm{C} 1$ \\
\hline Putinga (RS) & $\mathrm{A} 1$ & B1 & $\mathrm{C} 1$ \\
\hline Quaraí (RS) & $\mathrm{A} 2$ & B1 & $\mathrm{C} 1$ \\
\hline Quatro Irmãos (RS) & $\mathrm{A} 1$ & B2 & $\mathrm{C3}$ \\
\hline Quevedos (RS) & $\mathrm{A} 1$ & B2 & $\mathrm{C3}$ \\
\hline Quinze de Novembro (RS) & $\mathrm{A} 2$ & B1 & $\mathrm{C} 1$ \\
\hline Redentora (RS) & $\mathrm{A} 3$ & B2 & $\mathrm{C} 4$ \\
\hline Relvado (RS) & $\mathrm{A} 1$ & B1 & $\mathrm{C} 1$ \\
\hline Restinga Seca (RS) & $\mathrm{A} 1$ & B1 & $\mathrm{C} 1$ \\
\hline Rio dos Índios (RS) & $\mathrm{A} 1$ & B2 & $\mathrm{C3}$ \\
\hline Rio Grande (RS) & $\mathrm{A} 2$ & B1 & $\mathrm{C} 1$ \\
\hline Rio Pardo (RS) & $\mathrm{A} 1$ & B1 & C1 \\
\hline Riozinho (RS) & $\mathrm{A} 1$ & B2 & $\mathrm{C} 1$ \\
\hline Roca Sales (RS) & $\mathrm{A} 2$ & B1 & $\mathrm{C} 1$ \\
\hline Rodeio Bonito (RS) & $\mathrm{A} 1$ & B1 & $\mathrm{C} 1$ \\
\hline Rolador (RS) & $\mathrm{A} 1$ & B2 & $\mathrm{C} 1$ \\
\hline Rolante (RS) & $\mathrm{A} 1$ & B1 & $\mathrm{C} 1$ \\
\hline Ronda Alta (RS) & $\mathrm{A} 1$ & B1 & $\mathrm{C} 1$ \\
\hline Rondinha (RS) & $\mathrm{A} 1$ & B1 & $\mathrm{C} 1$ \\
\hline Roque Gonzales (RS) & $\mathrm{A} 1$ & B2 & $\mathrm{C} 1$ \\
\hline Rosário do Sul (RS) & $\mathrm{A} 1$ & B1 & $\mathrm{C} 1$ \\
\hline Sagrada Família (RS) & $\mathrm{A} 1$ & B2 & $\mathrm{C} 3$ \\
\hline Saldanha Marinho (RS) & $\mathrm{A} 2$ & B1 & $\mathrm{C} 1$ \\
\hline Salto do Jacuí (RS) & $\mathrm{A} 1$ & B1 & $\mathrm{C} 1$ \\
\hline Salvador das Missões (RS) & $\mathrm{A} 2$ & B1 & $\mathrm{C} 1$ \\
\hline Salvador do Sul (RS) & $\mathrm{A} 2$ & B1 & $\mathrm{C} 1$ \\
\hline Sananduva (RS) & $\mathrm{A} 1$ & B1 & $\mathrm{C} 1$ \\
\hline Santa Bárbara do Sul (RS) & $\mathrm{A} 1$ & $\mathrm{~B} 1$ & $\mathrm{C} 1$ \\
\hline
\end{tabular}




\begin{tabular}{|c|c|c|c|}
\hline Santa Cecília do Sul (RS) & A1 & B1 & C1 \\
\hline Santa Clara do Sul (RS) & $\mathrm{A} 1$ & B1 & C1 \\
\hline Santa Cruz do Sul (RS) & $\mathrm{A} 2$ & B1 & $\mathrm{C} 1$ \\
\hline Santa Margarida do Sul (RS) & A1 & B2 & $\mathrm{C} 3$ \\
\hline Santa Maria do Herval (RS) & $\mathrm{A} 2$ & B1 & $\mathrm{C} 1$ \\
\hline Santa Maria (RS) & $\mathrm{A} 2$ & B3 & $\mathrm{C} 1$ \\
\hline Santana da Boa Vista (RS) & $\mathrm{A} 1$ & B2 & $\mathrm{C} 3$ \\
\hline Sant'Ana do Livramento (RS) & $\mathrm{A} 2$ & B1 & C1 \\
\hline Santa Rosa (RS) & $\mathrm{A} 2$ & B1 & C1 \\
\hline Santa Tereza (RS) & $\mathrm{A} 2$ & B1 & C1 \\
\hline Santa Vitória do Palmar (RS) & A1 & B1 & $\mathrm{C} 1$ \\
\hline Santiago (RS) & $\mathrm{A} 1$ & B1 & $\mathrm{C} 1$ \\
\hline Santo Ângelo (RS) & $\mathrm{A} 2$ & B1 & C1 \\
\hline Santo Antônio da Patrulha (RS) & $\mathrm{A} 1$ & B1 & C1 \\
\hline Santo Antônio das Missões (RS) & $\mathrm{A} 1$ & B2 & $\mathrm{C} 3$ \\
\hline Santo Antônio do Palma (RS) & A1 & B1 & $\mathrm{C} 1$ \\
\hline Santo Antônio do Planalto (RS) & A1 & B1 & $\mathrm{C} 1$ \\
\hline Santo Augusto (RS) & $\mathrm{A} 1$ & B1 & C1 \\
\hline Santo Cristo (RS) & $\mathrm{A} 2$ & B1 & C1 \\
\hline Santo Expedito do Sul (RS) & A1 & B1 & $\mathrm{C} 1$ \\
\hline São Borja (RS) & A1 & B1 & C1 \\
\hline São Domingos do Sul (RS) & $\mathrm{A} 2$ & B1 & $\mathrm{C} 1$ \\
\hline São Francisco de Assis (RS) & $\mathrm{A} 1$ & B2 & $\mathrm{C} 3$ \\
\hline São Francisco de Paula (RS) & $\mathrm{A} 1$ & B1 & C1 \\
\hline São Gabriel (RS) & $\mathrm{A} 1$ & B1 & $\mathrm{C} 1$ \\
\hline São Jerônimo (RS) & A1 & B1 & $\mathrm{C} 1$ \\
\hline São João da Urtiga (RS) & A1 & B1 & $\mathrm{C} 1$ \\
\hline São João do Polêsine (RS) & A1 & B1 & $\mathrm{C} 1$ \\
\hline São Jorge (RS) & $\mathrm{A} 1$ & B1 & C1 \\
\hline São José das Missões (RS) & $\mathrm{A} 1$ & B2 & $\mathrm{C} 3$ \\
\hline São José do Herval (RS) & A1 & B2 & $\mathrm{C} 4$ \\
\hline São José do Hortêncio (RS) & $\mathrm{A} 2$ & B1 & C1 \\
\hline São José do Inhacorá (RS) & $\mathrm{A} 2$ & B1 & $\mathrm{C} 1$ \\
\hline São José do Norte (RS) & A3 & B2 & $\mathrm{C} 4$ \\
\hline São José do Ouro (RS) & $\mathrm{A} 1$ & B1 & C1 \\
\hline São José dos Ausentes (RS) & $\mathrm{A} 1$ & B1 & $\mathrm{C} 1$ \\
\hline São José do Sul (RS) & $\mathrm{A} 2$ & B1 & C1 \\
\hline São Leopoldo (RS) & $\mathrm{A} 2$ & B1 & $\mathrm{C} 1$ \\
\hline São Lourenço do Sul (RS) & A1 & B1 & $\mathrm{C} 1$ \\
\hline São Luiz Gonzaga (RS) & $\mathrm{A} 1$ & B1 & C1 \\
\hline São Marcos (RS) & $\mathrm{A} 2$ & B1 & C1 \\
\hline São Martinho da Serra (RS) & $\mathrm{A} 1$ & B2 & $\mathrm{C} 3$ \\
\hline São Martinho (RS) & $\mathrm{A} 1$ & B1 & C1 \\
\hline São Miguel das Missões (RS) & $\mathrm{A} 1$ & B1 & $\mathrm{C} 3$ \\
\hline São Nicolau (RS) & $\mathrm{A} 1$ & B2 & $\mathrm{C} 3$ \\
\hline São Paulo das Missões (RS) & $\mathrm{A} 1$ & B1 & C1 \\
\hline São Pedro da Serra (RS) & $\mathrm{A} 2$ & B1 & C1 \\
\hline São Pedro das Missões (RS) & $\mathrm{A} 1$ & B2 & $\mathrm{C} 3$ \\
\hline São Pedro do Butiá (RS) & $\mathrm{A} 2$ & B1 & $\mathrm{C} 1$ \\
\hline São Pedro do Sul (RS) & A1 & B1 & $\mathrm{C} 1$ \\
\hline São Sebastião do Caí (RS) & $\mathrm{A} 2$ & B1 & C1 \\
\hline São Sepé (RS) & $\mathrm{A} 1$ & $\mathrm{~B} 1$ & C1 \\
\hline
\end{tabular}




\begin{tabular}{|c|c|c|c|}
\hline São Valentim do Sul (RS) & $\mathrm{A} 2$ & B1 & C1 \\
\hline São Valentim (RS) & A1 & B2 & $\mathrm{C} 1$ \\
\hline São Valério do Sul (RS) & A1 & B2 & $\mathrm{C} 3$ \\
\hline São Vendelino (RS) & $\mathrm{A} 2$ & B1 & $\mathrm{C} 1$ \\
\hline São Vicente do Sul (RS) & A1 & B1 & $\mathrm{C} 1$ \\
\hline Sapiranga (RS) & $\mathrm{A} 2$ & B1 & C1 \\
\hline Sapucaia do Sul (RS) & $\mathrm{A} 2$ & B1 & C1 \\
\hline Sarandi (RS) & $\mathrm{A} 1$ & B1 & C1 \\
\hline Seberi (RS) & $\mathrm{A} 1$ & $\mathrm{~B} 2$ & C1 \\
\hline Sede Nova (RS) & $\mathrm{A} 1$ & B2 & $\mathrm{C} 1$ \\
\hline Segredo (RS) & $\mathrm{A} 1$ & B2 & $\mathrm{C} 3$ \\
\hline Selbach (RS) & $\mathrm{A} 2$ & B3 & C1 \\
\hline Senador Salgado Filho (RS) & A1 & B1 & C1 \\
\hline Sentinela do Sul (RS) & $\mathrm{A} 1$ & B2 & C1 \\
\hline Serafina Corrêa (RS) & $\mathrm{A} 2$ & B1 & $\mathrm{C} 1$ \\
\hline Sério (RS) & $\mathrm{A} 1$ & B2 & $\mathrm{C3}$ \\
\hline Sertão (RS) & A1 & B1 & C1 \\
\hline Sertão Santana (RS) & $\mathrm{A} 1$ & B1 & $\mathrm{C} 3$ \\
\hline Sete de Setembro (RS) & $\mathrm{A} 1$ & B1 & $\mathrm{C} 1$ \\
\hline Severiano de Almeida (RS) & $\mathrm{A} 1$ & B1 & C1 \\
\hline Silveira Martins (RS) & $\mathrm{A} 1$ & B1 & $\mathrm{C} 1$ \\
\hline Sinimbu (RS) & A1 & B1 & $\mathrm{C} 3$ \\
\hline Sobradinho (RS) & $\mathrm{A} 1$ & B1 & C1 \\
\hline Soledade (RS) & $\mathrm{A} 1$ & B1 & $\mathrm{C} 1$ \\
\hline Tabaí (RS) & $\mathrm{A} 1$ & B1 & C1 \\
\hline Tapejara (RS) & $\mathrm{A} 1$ & B1 & $\mathrm{C} 1$ \\
\hline Tapera (RS) & $\mathrm{A} 2$ & B1 & $\mathrm{C} 1$ \\
\hline Tapes (RS) & $\mathrm{A} 1$ & B1 & C1 \\
\hline Taquara (RS) & $\mathrm{A} 2$ & B1 & C1 \\
\hline Taquari (RS) & $\mathrm{A} 1$ & B1 & $\mathrm{C} 1$ \\
\hline Taquaruçu do Sul (RS) & $\mathrm{A} 1$ & B2 & C1 \\
\hline Tavares (RS) & $\mathrm{A} 1$ & B2 & $\mathrm{C3}$ \\
\hline Tenente Portela (RS) & $\mathrm{A} 1$ & B2 & $\mathrm{C} 3$ \\
\hline Terra de Areia (RS) & $\mathrm{A} 1$ & B1 & $\mathrm{C} 1$ \\
\hline Teutônia (RS) & $\mathrm{A} 2$ & B1 & C1 \\
\hline Tio Hugo (RS) & $\mathrm{A} 1$ & B1 & $\mathrm{C} 1$ \\
\hline Tiradentes do Sul (RS) & $\mathrm{A} 1$ & B2 & $\mathrm{C3}$ \\
\hline Toropi (RS) & $\mathrm{A} 1$ & B1 & $\mathrm{C} 1$ \\
\hline Torres (RS) & $\mathrm{A} 2$ & B1 & C1 \\
\hline Tramandaí (RS) & $\mathrm{A} 1$ & B1 & C1 \\
\hline Travesseiro (RS) & $\mathrm{A} 2$ & $\mathrm{~B} 1$ & C1 \\
\hline Três Arroios (RS) & $\mathrm{A} 2$ & B1 & $\mathrm{C} 2$ \\
\hline Três Cachoeiras (RS) & $\mathrm{A} 2$ & B1 & $\mathrm{C} 1$ \\
\hline Três Coroas (RS) & $\mathrm{A} 1$ & B1 & $\mathrm{C} 1$ \\
\hline Três de Maio (RS) & $\mathrm{A} 1$ & B1 & C1 \\
\hline Três Forquilhas (RS) & $\mathrm{A} 1$ & B2 & $\mathrm{C} 3$ \\
\hline Três Palmeiras (RS) & $\mathrm{A} 1$ & B2 & $\mathrm{C} 3$ \\
\hline Três Passos (RS) & $\mathrm{A} 1$ & B1 & $\mathrm{C} 1$ \\
\hline Trindade do Sul (RS) & $\mathrm{A} 1$ & B2 & $\mathrm{C} 3$ \\
\hline Triunfo (RS) & $\mathrm{A} 1$ & B1 & C1 \\
\hline Tucunduva (RS) & $\mathrm{A} 2$ & B1 & C1 \\
\hline Tunas (RS) & $\mathrm{A} 3$ & $\mathrm{~B} 2$ & $\mathrm{C} 3$ \\
\hline
\end{tabular}




\begin{tabular}{|c|c|c|c|}
\hline Tupanci do Sul (RS) & $\mathrm{A} 1$ & B1 & $\mathrm{C3}$ \\
\hline Tupanciretã (RS) & $\mathrm{A} 1$ & B1 & $\mathrm{C} 1$ \\
\hline Tupandi (RS) & $\mathrm{A} 2$ & B1 & $\mathrm{C} 1$ \\
\hline Tuparendi (RS) & $\mathrm{A} 1$ & B1 & $\mathrm{C} 1$ \\
\hline Turuçu (RS) & $\mathrm{A} 1$ & B1 & $\mathrm{C} 1$ \\
\hline Ubiretama (RS) & $\mathrm{A} 2$ & B1 & $\mathrm{C} 1$ \\
\hline União da Serra (RS) & $\mathrm{A} 1$ & B1 & $\mathrm{C} 1$ \\
\hline Unistalda (RS) & $\mathrm{A} 1$ & B2 & $\mathrm{C} 3$ \\
\hline Uruguaiana (RS) & $\mathrm{A} 2$ & B1 & $\mathrm{C} 1$ \\
\hline Vacaria (RS) & $\mathrm{A} 1$ & B1 & $\mathrm{C} 1$ \\
\hline Vale do Sol (RS) & $\mathrm{A} 1$ & B1 & $\mathrm{C} 1$ \\
\hline Vale Real (RS) & $\mathrm{A} 2$ & B1 & $\mathrm{C} 1$ \\
\hline Vale Verde (RS) & $\mathrm{A} 1$ & B2 & $\mathrm{C} 3$ \\
\hline Vanini (RS) & $\mathrm{A} 1$ & B1 & $\mathrm{C} 1$ \\
\hline Venâncio Aires (RS) & $\mathrm{A} 1$ & B1 & $\mathrm{C} 1$ \\
\hline Vera Cruz (RS) & $\mathrm{A} 1$ & B1 & $\mathrm{C} 1$ \\
\hline Veranópolis (RS) & $\mathrm{A} 2$ & B3 & $\mathrm{C} 1$ \\
\hline Vespasiano Correa (RS) & $\mathrm{A} 1$ & B1 & $\mathrm{C} 1$ \\
\hline Viadutos (RS) & $\mathrm{A} 1$ & B1 & $\mathrm{C} 1$ \\
\hline Viamão (RS) & $\mathrm{A} 2$ & B1 & $\mathrm{C} 1$ \\
\hline Vicente Dutra (RS) & $\mathrm{A} 1$ & B2 & $\mathrm{C} 3$ \\
\hline Victor Graeff (RS) & $\mathrm{A} 1$ & B1 & $\mathrm{C} 1$ \\
\hline Vila Flores (RS) & $\mathrm{A} 2$ & B1 & $\mathrm{C} 1$ \\
\hline Vila Lângaro (RS) & $\mathrm{A} 1$ & B1 & $\mathrm{C} 1$ \\
\hline Vila Maria (RS) & $\mathrm{A} 1$ & B1 & $\mathrm{C} 1$ \\
\hline Vila Nova do Sul (RS) & $\mathrm{A} 1$ & B2 & $\mathrm{C3}$ \\
\hline Vista Alegre do Prata (RS) & $\mathrm{A} 2$ & B1 & $\mathrm{C} 1$ \\
\hline Vista Alegre (RS) & $\mathrm{A} 1$ & B1 & $\mathrm{C} 1$ \\
\hline Vista Gaúcha (RS) & $\mathrm{A} 1$ & B1 & $\mathrm{C} 1$ \\
\hline Vitória das Missões (RS) & $\mathrm{A} 1$ & $\mathrm{~B} 2$ & $\mathrm{C} 3$ \\
\hline Westfalia (RS) & $\mathrm{A} 2$ & B3 & $\mathrm{C} 1$ \\
\hline Xangri-Lá (RS) & $\mathrm{A} 2$ & $\mathrm{~B} 1$ & $\mathrm{C} 1$ \\
\hline
\end{tabular}

